\title{
The BCI as a Pervasive Technology - A Research Plan
}

\author{
C. P. Brennan \\ PhD Student, Smart \\ Environments \\ University of Ulster \\ Jordanstown, N. Ireland \\ brennan-c15 \\ @email.ulster.ac.uk
}

\author{
P. J. McCullagh \\ Computer Science \\ Research Institute \\ University of Ulster \\ Jordanstown, N. Ireland \\ pj.mccullagh \\ @ulster.ac.uk
}

\author{
G. Lightbody \\ Computer Science \\ Research Institute \\ University of Ulster \\ Jordanstown, N. Ireland \\ g.lightbody \\ @ulster.ac.uk
}

\author{
L. Galway \\ Computer Science \\ Research Institute \\ University of Ulster \\ Jordanstown, N. Ireland \\ I.galway \\ @ulster.ac.uk
}

\begin{abstract}
In this work we explore whether the BrainComputer Interface (BCI) can become a pervasive technology. The primary goal of BCI technology has been to provide communication and control for people with severe neural dysfunction, which affects their ability to interact with the environment. BCI research has focused largely on noninvasive, electroencephalography (EEG) based BCI methods. A number of EEG consumer grade devices are now available; the Emotiv EPOC neuroheadset is one such device. This paper investigates the utility, performance and usability of this device in an office setting by using EEG correlates of biosignals for control. The results suggest that it is possible for some users to achieve reasonable control with only minutes of training. However sustaining engagement over a longer duration and widespread use provide suitable areas for further investigation. As a contribution to the Doctoral Colloquium the paper provides an account of intended contributions of this BCI research, the plan of study necessary to achieve this goal, and enumerates the major research questions to be addressed in the next two years. The longerterm aim is to deploy BCI as a Pervasive technology.
\end{abstract}

Index Terms-Augmentation, Communication, BrainComputer Interface (BCI), Electroencephalography (EEG), Electrooculography (EOG), Emotiv EPOC, Pervasive.

\section{INTRODUCTION}

Over the past 30 years, the life expectancy of the population in developed countries has steadily risen. This extension in longevity significantly increases the number of people that are most susceptible to disease and disability [1], [5] and in need of assistive technology. Some disorders can disrupt the neuromuscular channels through which the brain communicates and controls its external environment [16]. These conditions include amyotrophic lateral sclerosis, multiple sclerosis, muscular dystrophies, brainstem stroke, and a number of other diseases. Although prevalence is very small the increased probability of contracting one of these conditions impacts the demand for innovation in the field of healthcare with technological advancements from a healthcare perspective. Thus assistive

Permission to make digital or hard copies of all or part of this work fora personal or classroom use is granted without fee provided that copies are not made or distributed for profit or commercial advantage and that copies bear this notice and the full citation on the first page. To copy otherwise, to republish, to post on servers or to redistribute to lists, requires prior specific permission and/or a fee. PervasiveHealth 2014, May 20-23, Oldenburg, Germany Copyright (C) 2014 ICST 978-1-63190-011-2 DOI 10.4108/icst.pervasivehealth.2014.255755 technology could greatly improve the quality of life and independence for those with a disorder of the aforementioned nature.

One such technology that offers this potential is that of BCI. A generally accepted definition of a BCI is a system that provides a communication channel for users, which does not rely on physical movement [12]. Additionally, these systems can be categorised as either invasive or non-invasive. The former refers to systems that require microelectrode arrays to be placed directly into the cortex in order to derive the user's intent from neuronal action potentials or local field potentials [15]. Whereas the latter derives the user's intent from scalp recorded EEG activity that does not require clinical procedures to implant the electrodes [15]. Due to the substantial technical difficulties and clinical risks associated with invasive BCIs, non-invasive methods are the only viable potential pervasive $\mathrm{BCI}$ technology.

Medical grade BCI devices use EEG to measure brain activity in order to determine user intent from which to control and/or communicate with devices and applications [7]. Whilst these devices can achieve this goal, applications suffer from various limitations such as inaccuracies, inconvenient setup, timeconsuming process, complex applications, and the need for carefully controlled environments [13]. Recent advancements have led to the release of consumer grade $\mathrm{BCI}$ devices that can potentially overcome some of these limitations. However there is a lack of evidence at the present time with regard to the utility of such commercial devices and their performance. One device of this kind is the Emotiv EPOC, which is a wireless and aesthetically pleasing neuroheadset that offers a multichannel EEG system. The device features a user-friendly software interface, a design that requires minimal experimenter assistance, and the ability to reduce electrode impedance by saline solution (as opposed to the gel needed for medical grade devices). Studies are now being reported that evaluate the performance of the Emotiv EPOC [10], [14]. Some studies have shown that this device may not be suitably accurate for critical applications [8], [9], but this requires further evidence. This study evaluates the Emotiv EPOC in terms of flexibility, performance and usability for healthy users. 


\section{Method}

The purpose of this study was to address a specific research question: Can a consumer grade BCI device (such as the EPOC) provide an acceptable EEG measure to function as part of a collaborative assistive technology?

Four subjects who do not suffer from brain injury participated in this study. Each participant gave informed consent for the study, which had been reviewed and approved by the Ethics Committee of the Faculty of Computing and Engineering in the University of Ulster.

\subsection{Preparation}

Each participant was required to take part in a short recording session that lasted no more than 1 hour inclusive of setup and data acquisition. Before this began, the Emotiv EPOC was cleaned and prepared for use. The cleaning procedure involved a $50 \%$ diluted solution of white vinegar and a soft cloth. The rear of each sensor was gently agitated with this solution to remove any corrosion. Before each trial, all electrodes and felt pads where placed in a hydrator pack and a saline solution applied to each. After this, each electrode was secured to the device and positioned appropriately on the head of the subject.

\subsection{Training}

At the beginning of the session the participant was required to undergo a training procedure facilitated by the Cognitiv Suite within the control panel application. The Cognitiv Suite employs various approaches such as EEG and electrooculography (EOG). It records and interprets a user's conscious EEG and intent so as to enable the user to manipulate virtual objects. This suite was used to train a 'neutral' state plus four additional commands left, right, lift, and drop. When training the neutral state the participant was required to relax, clear their thoughts and think of nothing in particular. To train the commands left and right the participant was asked to focus their gaze on markers to the left and right of the screen. To train the lift command the participant was required to clench their teeth and to train the drop command they were asked to tap their left foot. Each trial commenced only after the user had trained each command to an accuracy of greater than $70 \%$ (as advised by the Emotiv detection suite, Cognitiv). For all subjects each command had 3 to 15 training periods, each lasting 8 seconds.

\subsection{Experimental Design}

Once the session began, the participant was issued with twenty requests (e.g. move in one of four directions) and allowed ten seconds to complete each request. A five second rest period was given between each request in which the participant was asked to relax and simulate the neutral state. For each of the twenty requests the participant had to concentrate on moving an object to one of four locations on the screen top, bottom, left, or right. In order move the object right or left the user was required to focus their gaze on a marker located to the left or right of the screen. In order to move the object up the user was required to clench their teeth and to move it down the user was required to tap their left foot [Figure 1].

Correctly completed requests were assigned a value of 1 and incorrectly completed requests were assigned a value of 0 . This allowed for an accuracy value to be determined. Including the training phase each session took no longer than 30 minutes to complete.

Curtailing a recording session to half an hour contrasts with normal laboratory based recording, where set up time for electrode preparation and placement may take 15-20 minutes and cleaning time may add another five, yielding a total session time in excess of one hour. The easy to use interface with real time feedback on the state of electrodes also improves usability, particularly where non-experts are involved. This has significant impact on the user experience.

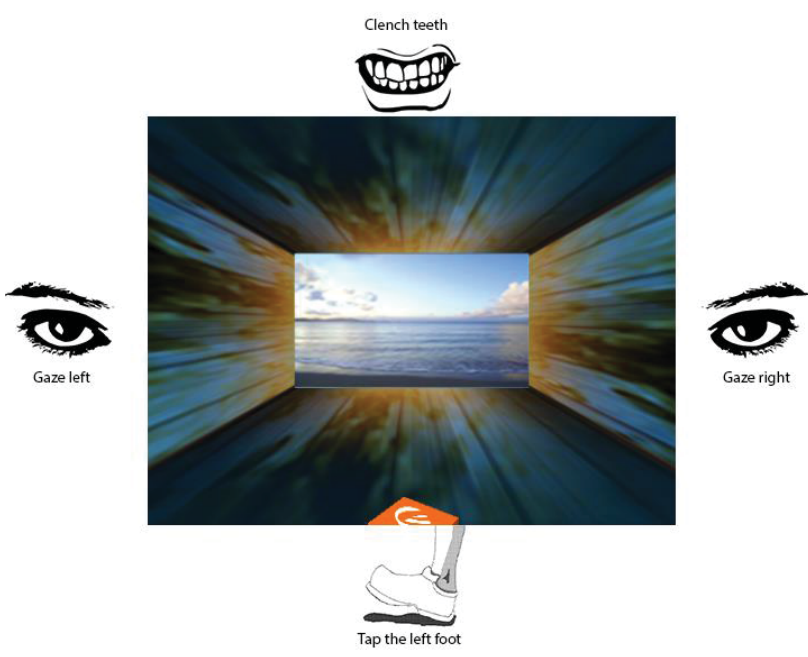

Figure 1. An illustration of the control paradigm. In this example the user is sucessfully replicating the drop command by tapping their left foot.

\section{RESULTS}

Over the initial training phases all four subjects acquired a reported skill level greater than $70 \%$ for each command. Table 1 displays the overall skill rating for all subjects. Furthermore, it defines the skill rating of each individual command for all participants. In addition to this, Table 2 presents the actual accuracy and defines the number of each request that was completed correctly. Each subject exceeded the $20 \%$ accuracy expected by chance. The mean accuracy for all subjects equates to $64 \%$ with subjects B and C performing greater than $85 \%$. Each of the four commands was issued five times per participant in a stochastic order. All subjects were able to correctly complete the up command $100 \%$ of the time, the right command $55 \%$ of the time and the left and down commands $50 \%$ of the time. However some subjects performed significantly better than others indicating the inter subject variability.

Subjects A and D were unable to perform the down command successfully. The down command requires a degree of physical movement and this may disrupt the EEG signal by introducing unpredictable movement artefacts. It is possible that the vibrations caused from tapping of the foot created noise in the scalp recorded EEG.

The second least successful command was left since subject D could not complete it at all and subject A could only complete it once. Observation showed that inaccurate control of this command may be due to a difference in training and replication strategies, i.e. the subject trained the command on the correlated brain signal for eye movement but during the trial attempted to control the left command by turning their head slightly to focus on the target. As both left and right commands are controlled by EOG, the software may have difficulties differentiating between the two, as a movement to the right is always followed by a return to the left, introducing the need for more considered synchronisation. Nevertheless, further investigation is required to 
achieve more conclusive results and provide a potential solution to the problem.

Table 1- Training Skill Rating

\begin{tabular}{ccccccc}
\hline Subject & Sex & $\begin{array}{c}\text { Overall } \\
\text { Skill } \\
\text { Rating }\end{array}$ & Left & Right & Lift & Drop \\
\hline A & M & $83 \%$ & $86 \%$ & $94 \%$ & $76 \%$ & $76 \%$ \\
B & M & $79 \%$ & $77 \%$ & $71 \%$ & $91 \%$ & $78 \%$ \\
C & M & $81 \%$ & $74 \%$ & $83 \%$ & $87 \%$ & $80 \%$ \\
D & F & $81 \%$ & $80 \%$ & $95 \%$ & $71 \%$ & $78 \%$ \\
& Mean: & $\mathbf{8 1 \%}$ & $\mathbf{7 9 \%}$ & $\mathbf{8 6 \%}$ & $\mathbf{8 1 \%}$ & $\mathbf{7 8 \%}$
\end{tabular}

Table 2- Subject Accuracy for the Test

\begin{tabular}{ccccccc}
\hline & \multicolumn{5}{c}{ Requests Completed Correctly } \\
\hline Subject & Sex & $\begin{array}{c}\text { Actual } \\
\text { Accuracy }\end{array}$ & Left & Right & U & Down \\
\hline A & M & $35 \%$ & 1 & 1 & 5 & 0 \\
B & M & $85 \%$ & 4 & 3 & 5 & 5 \\
C & M & $90 \%$ & 5 & 3 & 5 & 5 \\
D & F & $45 \%$ & 0 & 4 & 5 & 0 \\
& Total: & $\mathbf{6 4 \%}$ & $\mathbf{1 0}$ & $\mathbf{1 1}$ & $\mathbf{2 0}$ & $\mathbf{1 0}$
\end{tabular}

\section{DISCUSSION AND CONCLUSION}

This paper describes the use of a consumer grade BCI device, the Emotiv EPOC for manipulating a virtual object based on eye gaze direction and actual movement. The results show that accurate control can be achieved with little training. Participants that possessed more BCI experience seemed to perform better. While this is a preliminary study, it suggests that the accuracy is not dependent on the overall skill rating as reported by the Cognitiv Suite. All four subjects acquired differing degrees of control with subjects A and D performing less well, yet subject A had the highest overall skill rating.

It was not possible to determine how the EPOC compares to a medical grade device in terms of performance and such work needs to be undertaken to provide further benchmarks. There are a number of previous studies that suggest that the EPOC performance is lower than that of a medical grade BCI [8], [14]. However these studies do not address usability and flexibility [2], [8] and it is clear from initial feedback that the EPOC was readily accepted, and better in terms of usability.

All participants had experience of medical grade devices and stated that the EPOC is much more comfortable and less difficult to setup. One subject indicated that the CMS and DRL references were the most uncomfortable part of the interaction. All subjects agreed that, as with any $\mathrm{BCI}$ device, prolonged use causes fatigue.

It is evident that further investigations are required to achieve more conclusive results. However this preliminary study demonstrates that specific users are able to gain accurate control with little effort, though suggests that this will not be the case for all users. Some studies have shown that healthy participants are able to gain better control of BCI systems than severely disabled participants [12]. Consequently, if an adequate level of control can be achieved with a device of this nature then it may be possible to complement and improve performance with a completely separate technology (e.g. Eye tracking). If this is the case, less accurate and inconsistent consumer grade BCI devices can play a fundamental role in the evolution of $\mathrm{BCI}$ and hybrid systems.

\section{FUTURE WORK}

The study undertaken here points to four areas that should be further explored: a larger number of subjects, comparison with laboratory BCI, other control paradigms and hybrid systems.

- By performing the same experiment with a larger number of subjects $(\mathrm{N}>50)$ more conclusive results can be achieved with regard to widespread BCI ability and demographics.

- Further comparison with medical grade devices is needed. The systems differ in terms of accuracy, set up and cost. However it may be possible to demonstrate that EPOC has sufficient flexibility and accuracy to achieve widespread deployment, as accuracy may be matched to intended application.

- This study used 'commands' which are easy to reproduce (foot tapping, left and right eye movement and teeth clenching). The experiment can be conducted again with a different control paradigm. For example actual movement could be replaced with 'imagined' movement as this aligns with application for users with more severe deficits. It is recognised that this paradigm is more difficult to master; therefore the training time will subsequently be increased.

- Focus on the EPOC as a component of a hybrid BCI (hBCI) would potentially provide more robust performance and ameliorate any deterioration in accuracy when compared with medical grade systems.

Thus a number of issues still need to be addressed and potentially solved. Previous and contemporary research studies have focused on BCI for non-critical applications such as gaming [4], [6], [11]. Such technology will be readily available at an affordable price (less than 500 Euro) and therefore it could become a technology for all potential users. Indeed an improved hBCI system would further expand the target market for multimodal human computer interaction. It is, however clear that in a BCI, the user (brain) has the final say in determining whether such devices can be accepted and finally become pervasive.

\section{Ph.D. Research}

This section emphasises the Ph.D. plan of study, potential research contributions, and major research questions. The Ph.D. proposal focuses on BCI and eye tracking technology as a Collaborative Assistive Technology. The primary aim is to support ongoing research and develop an effective hBCI system.

\subsection{Plan of Study}

Within this research endeavour, a series of planned activities can be identified. It was a necessity to conduct extensive research in order to gain substantial knowledge within the field of BCI, assistive technology and hybrid systems. This research has and will continue to provide experience with data analysis tools and software development kits (SDK) such as OpenVibe, MATLAB, EEGLAB, BCILAB and the Emotiv framework etc. The next planned activity is to begin developing an hBCI system that can improve upon current systems in terms of performance, reliability and usability. After which, it is necessary to conduct a number of studies.

These studies aim to identify the feasibility of commercial BCI devices for the hybrid approach, widespread BCI ability and demographics, efficiency of different BCI paradigms and the improvements of combining multiple BCI paradigms with separate technologies. The majority of existing research is performed on healthy subjects under laboratory conditions rather than on target user groups in domestic settings. The final studies 
of this proposal will aim to engage with community partners in the Cedar Foundation in order to evaluate the developed hybrid technology on people with brain injury thus providing early access of this emerging assistive technology to those who potentially need it the most.

\subsection{Research Contributions}

A research contribution to computing science is possible within multiple capacities: (i) the development of a hybrid architecture, (ii) data analysis and processing improvements, (iii) graphical user interface design for hybrid systems, and (iv) high frequency steady state visually evoked potential (SSVEP) protocols.

The development of an effective hybrid architecture could have a substantial impact in the field of BCI. While it is evident that BCI technology experiences various limitations including performance, usability and reliability constraints, these limitations can be overcome by a hybrid approach. An approach that combines a multitude of BCI paradigms (e.g. an active motor imagery protocol coupled with a reactive SSVEP protocol) with separate input modalities (e.g. eye tracking). Preliminary studies suggest that $\mathrm{hBCI}$ is an innovative and viable solution to facilitate the evolution of $\mathrm{BCI}$ technology across multidisciplinary application areas including 'communication and control', 'entertainment', 'motor recovery', and 'motor substitution'.

Additionally, it is likely that data analysis techniques can be employed to determine features appropriate to improved classification for the hybrid approach. Eye tracking systems can provide efficient navigation (e.g. control of a cursor) but are limited by intentional selection commands (e.g. mouse click); an area of significant attention in BCI. When measuring EEG activity, other correlates of bio-signal can be identified that are representative of other input modalities. It may be possible to extract features from the EEG that represent the eye tracking data. For example using dwells from eye tracking in an attempt to identify an intended selection in the EEG and distinguish this from other types of dwell such as pausing to read or losing concentration.

A research contribution is also possible in the form of graphical user interface design for the hybrid approach. The collaboration of the inputs from BCI and eye tracking modalities, which may have different temporal properties, can be utilised to interact with a menu system and provide actuation of outputs. Such a system would need to be designed and developed without the use of existing structures, documents, and procedures. To our knowledge this type of work is yet to be undertaken thus ensuring its originality and contribution to knowledge.

Finally, it is possible to make a research contribution towards $\mathrm{BCI}$ as a pervasive technology. As the majority of SSVEP based BCI studies have focused on low frequency protocols, which suffer from photosensitivity concerns, a substantial barrier preventing SSVEP methods from becoming pervasive exists. Therefore an investigation into high frequency SSVEP is feasible. An SSVEP protocol consisting of a frequency above $35 \mathrm{~Hz}$, the lower limit of the flicker fusion threshold (a concept that defines the frequency at which an intermittent visual stimulus appears to be steady to the human observer) may prove to be the solution.

\subsection{Research Questions}

Extensive research has been conducted in which a number of research question have been identified:

1. Can consumer grade BCI devices (such as the Emotiv EPOC) provide an acceptable EEG measure to function as part of a collaborative assistive technology?

2. What architecture would constitute a more robust hBCI system? Which BCI paradigms should be combined with eye tracking technology to provide an efficient level of communication and control?

3. Can the collaboration of eye tracking and EEG data be used to determine intended selection?

4. Is it possible to use the combination of EEG and eye tracking data to identify error potentials and autonomously correct false positive no-control states?

5. Can this technology become pervasive?

\section{REFERENCES}

[1] Allison, B.Z, "Future BNCI: A Roadmap for Future Directions in Brain / Neuronal Computer Interaction Research," http://future-bnci.org/images/ stories/Future BNCI Roadmap.pdf (accessed September 2013) 1 1. (2012), 1-255.

[2] Badcock, N. a et al. 2013. Validation of the Emotiv EPOC EEG gaming system for measuring research quality auditory ERPs. PeerJ. 1, (2013), e38.

[3] Biosemi. 2013. What is the function of CMS and DRL electrodes Ground, Reference or what? (2013). Retrieved December 1, 2013 from http://www.biosemi.com/faq/cms\&drl.htm

[4] Bos, D.O. and Reuderink, B. 2008. BrainBasher: a BCI Game. Methods. (2008), 7-10.

[5] Christensen, K. et al. 2009. Ageing populations: the challenges ahead. Lancet. 374, 9696 (Oct. 2009), 1196-208.

[6] Chumerin, N. et al. 2013. Steady-State Visual Evoked Potential-Based Computer Gaming on a Consumer-Grade EEG Device. 5, 2 (2013), 100-110.

[7] Duvinage, et al. 2013 "Performance of the Emotiv Epoc headset for P300-based applications," Biomedical engineering online, 12, (Jan. 2013), 56.

[8] Duvinage, M. et al. 2012. A P300-based Quantitative Comparison between the Emotiv Epoc Headset and a Medical EEG Device. Biomedical Engineering / 765: Telehealth / 766: Assistive Technologies. (2012).

[9] Duvinage, M. et al. 2013. Performance of the Emotiv Epoc headset for P300-based applications. Biomedical engineering online. 12, (Jan. 2013), 56.

[10] Ekanayake, H. 2010. P300 and Emotiv EPOC : Does Emotiv EPOC capture real EEG (2010), Retrieved from http://neurofeedback.visaduma.info/emotivresearch.htm,

[11] Marshall, D. et al. 2013. Games, Gameplay, and BCI : The State of the Art. 5, 2 (2013), 82-99.

[12] Mccullagh, P. et al. 2013. Investigation into a Mixed Hybrid Using SSVEP and Eye Gaze for Optimising User Interaction within a Virtual Environment. (2013), 530-539.

[13] Mulvenna, M. et al. 2012. Realistic expectations with brain computer interfaces. Journal of Assistive Technologies. 6, 4 (2012), 233-244.

[14] Stytsenko, K. et al (2011). Evaluation of consumer EEG device Emotiv EPOC. MEi: CogSci Conference 2011 Ljubljana. (2011). Retrieved from http://www.univie.ac.at/meicogsci/php/ocs/index.php/meicog /meicog2011/paper/view/210.

[15] Wolpaw, J.R. and McFarland, D.J. 2004. Control of a twodimensional movement signal by a noninvasive braincomputer interface in humans. Proceedings of the National Academy of Sciences of the United States of America. 101, 51 (Dec. 2004), 17849-54.

[16] Wolpaw, J.R. et al. 2002. Brain - computer interfaces for communication and control. Clinical Neurophysiology. 113, 6 (2002), 767-791. 